\title{
Dentre as mais complexas tecnologias em saúde, o medicamento pode matar ou salvar vidas
}

Maurilio de Souza Cazarim¹, Aílson da Luz André de Araujo ${ }^{1}$

A Revista Medicina (Ribeirão Preto) publica nesta edição dois artigos que abordam um tema de suma importância para a morbimortalidade ocasionada por medicamentos, intoxicação causada por medicamentos e interações medicamentosas.

Os riscos à saúde causados pelo manejo ineficiente da farmacoterapia ou pelo uso inadequado dos medicamentos são fatores reconhecidos como os principais responsáveis pelos altos índices de Problemas Relacionados à Farmacoterapia (PRFs) e iatrogenias, o que pode ser causa de morte e elevar custos aos sistemas de saúde ${ }^{1}$.

Ressalta-se que o Uso Racional de Medicamentos, definido pela Organização Mundial da Saúde em 1985 e, que vigora até os dias atuais, predita que²:

"os pacientes recebam medicamentos adequados às suas necessidades clínicas, em doses que atendam às suas necessidades individuais, por um período de tempo adequado e ao menor custo para eles e sua comunidade".

O contexto da pandemia por COVID-19, doença emergente causada pelo vírus SARS-Cov-2, da família coronavírus, mostrou ser necessário um novo olhar para a saúde, principalmente vindo de Instituições governamentais e sociedade, as quais devem atentar-se às evidências em saúde e ao uso racional de medicamentos. Haja vista, o destaque nos noticiários pela busca desenfreada por tratamentos ultrapassando, algumas vezes, os preceitos éticos e minimizando a saúde baseada em evidências e o uso abusivo de medicamentos pela sociedade na tentativa de se prevenir e/ou curar-se.

As consequências podem ir além dos problemas oriundos pelo uso inadequado do medicamento, tais como as reações adversas, interações e intoxicações medicamentosas, pois acabam por comprometer o abastecimento de unidades farmacêuticas e, consequentemente, o acesso aos tratamentos preconizados em suas indicações originais de registro para doenças específicas, ocasionando o comprometimento ao assistencialismo na saúde.

De acordo com os dados do Sistema Nacional de Informações Tóxico-Farmacológicas (SINITOX), uma das maiores causas de intoxicações no Brasil é o uso inadequado ou abusivo de medicamentos. Os números mostram que dos 19,5 milhões de pessoas internadas no Brasil no ano de 2017, aproximadamente 1,3 milhões pacientes apresentaram ao menos um problema relacionado ao uso do medicamento durante seu tratamento. Dentre os eventos adversos oriundos de falhas no processo do uso do medicamento, aqueles que foram considerados graves corresponderam a 54.769 óbitos, sendo que 36.174 eram preveníeis ${ }^{3}$.

O artigo "Internações por intoxicações medicamentosas em crianças menores de cinco anos no estado de Minas Gerais/Brasil, 2009 - 2018" trata-se de um estudo descritivo analítico de levantamento, cujo tema é de muita relevância ao cenário clínico e toxicológico, bem como para a abrangência de políticas de saúde voltadas ao uso racional de medicamentos. Os autores descrevem internações hospitalares no estado de Minas Gerais devido às intoxicações medicamentosas ao longo de nove anos ${ }^{4}$.

Departamento de Ciências Farmacêuticas, Faculdade de Farmácia, Universidade Federal de Juiz de Fora, campus Juiz de Fora-MG, Brasil.

Maurilio de Souza Cazarim, Faculdade de Farmácia, Universidade Federal de Juiz de Fora (UFJF). Rua José Lourenço Kelmer, s/n - Campus Universitário, Bairro São Pedro. CEP: 36036-900. Juiz de Fora (MG), Brasil.

maurilio.cazarim@ufjf.br 
Neste âmbito, o artigo mostra, com os dados levantados por aproximadamente 12 milhões de registros do sistema nacional DATASUS Tabwin, que as classes terapêuticas mais frequentes, depois dos fármacos que não foram especificados nos registros de intoxicações, foram os antiepilépticos/ sedativo-hipnóticos/antiparkinsonianos $(14,72 \%)$; fármacos psicotrópicos $(6,62 \%)$. Os antibióticos sistêmicos apareceram com $4,88 \%$, o que revela uma alçada importante quanto ao uso racional de antimicrobianos e a possibilidade do aumento da resistência bacteriana no cenário nacional.

O artigo destaca a vulnerabilidade da população de faixa etária menor que cinco anos no que tange ao diferente metabolismo para o manejo de medicamentos (interações medicamentosas, erros na administração, prescrição inadequada de medicamentos, dificuldades com cálculo e medidas de dose); a característica exploratória que é marcante desta faixa etária e o risco das farmácias caseiras; a representação midiática do medicamento com a utilização de embalagens atraentes e medicamentos com sabor e em diferentes formatos, o que torna o caráter exploratório das crianças ainda mais aguçado.

Nesse sentido os autores discutem medidas capazes de reduzir o impacto destas internações, tais como: limitar o acesso de crianças aos medicamentos; apropriar locais seguros para o armazenamento dos medicamentos; adotar o uso de embalagens especiais e o fracionamento de doses, como previsto na Resolução da Diretoria Colegiada no 80 de 11 de maio de 2006, da Agência Nacional de Vigilância Sanitária (ANVISA) e; eles destacam o importante papel do farmacêutico na promoção da educação em saúde, orientação sobre o uso correto dos medicamentos. De fato são medidas importantes que podem fomentar, juntamente com os resultados apresentados, reformulações nas políticas públicas de saúde para a redução do impacto clínico e econômico causado pelas intoxicações medicamentosas.

No artigo "Fatores associados para potenciais interações medicamentosas clinicamente significantes em terapia intensiva adulto", os autores apresentaram os resultados de um estudo transversal 309 pacientes internados em uma unidade de terapia intensiva adulto de um hospital do centro oeste de Minas Gerais, Brasil5. Foram caracterizadas as interações medicamentosas potenciais (IMp) maiores e contraindicadas, segundo a classificação da base Micromedex 2.0, em terapia intensiva adulto e determinar sua prevalência, medicamentos e fatores associados à exposição dessas interações.

Destaca-se a contribuição dessa pesquisa a um cenário pobre em informações na literatura científica, a investigação da prevalência de IMp clinicamente significante em pacientes hospitalizados em UTI adulto no cenário nacional, além de identificar quais medicamentos colaboraram para elevar o número destas IMp e apresentar as principais implicações clínicas potencias que podem ser desencadeadas.

Os achados do estudo revelaram uma alta frequência $(81,8 \%)$ da amostra que apresentaram pelo menos uma IMp maior ou contraindicada, sendo que pessoas idosas, do sexo masculino e polimedicadas foram as mais expostas, corroborando estudos anteriores ${ }^{6,7}$. Foi possível evidenciar que os medicamentos mais envolvidos nas IMp continham fármacos comuns, com ação no sistema nervoso, como metoclopramida e dipirona. As implicações clínicas potenciais também foram identificadas, com destaque para o aumento dos riscos de reações extrapiramidais, depressão do sistema nervoso central e sangramento.

Os autores alertam para as dificuldades no manejo clínico das interações medicamentosas devido aos fatores de risco individuais não modificáveis. Além disso, os clínicos frequentemente ignoram ou subestimam interações severas disponíveis nos alertas eletrônicos ${ }^{8}$. Assim, os autores recomendam a participação dos diversos atores envolvidos no sistema de medicação como enfermeiros, farmacêuticos clínicos e médicos, para monitorar e identificar as IMp clinicamente relevantes, como forma de prevenir eventos adversos e melhorar a segurança do paciente; também o uso de prescrições eletrônicas com um sistema de alerta e bancos de dados para identificação de IMp, que podem permitir o rastreamento em tempo real de interações medicamentosas.

Embora haja limitações como a subnotificação e a fragilidade dos dados a serem coletados na Autorização de Internação Hospitalar quanto à dificuldade de identificar a correta atribuição do agente causador da intoxicação medicamentosa; a ausência de avaliação clínica para detecção da ocorrência da IMp; ausência de entrevistas com pacientes e familiares para identificar a prática de 
automedicação ou tratamentos alternativos, os estudos em destaque apresentam métodos coerentes ao que se propuseram a realizar e apresentam conclusões contundentes à realidade da morbi-mortalidade ocasionada pelos medicamentos.

\section{REFERÊNCIAS}

1. 1. Cazarim MS, Rodrigues JPV, Calcini PS, Einarson TR, Pereira LRL. Cost-benefit analysis of pharmacist interventions over 36 months in a university hospital. Rev Saude Publica. 2020;54:94.

2. Brasil. (2017). Fiocruz. Casos Registrados de Intoxicação Humana por Agente Tóxico e Circunstância. Sistema Nacional de Informações Tóxico-Farmacológicas (SINITOX). Rio de Janeiro: Fundação Oswaldo Cruz. Fiocruz. 2016. Disponível em: https://sinitox.icict.fiocruz.br/sites/sinitox.icict.fiocruz.br/files//Brasil6_1.pdf

3. World Health Organization (WHO). (1985). Expert Conference, 1985, Nairobi. Rational Use of Medicines. Report of the Expert Conference, Nairobi, 25-29 November 1985. Geneva: World Health Organization. 1986. 304 p. Disponível em: http://apps.who.int/ medicinedocs/documents/s17054e/s17054e.pdf
4. Bego BS, Pereira ML, Nogueira LS. Internações por intoxicações medicamentosas em crianças menores de cinco anos no estado de Minas Gerais/Brasil, 2009 - 2018. Medicina (Ribeirão Preto). 2020;53(4):370-378. doi: https://doi.org/10.11606/issn.2176-7262.v53i4p370-378

5. Moraes JT, Maia JM, Trindade OM, Oliveira LA, Sanches C, Trevisan DD. Fatores associados para potenciais interações medicamentosas clinicamente significantes em terapia intensiva adulto. Medicina (Ribeirão Preto). 2020;53(4):379-388. doi: https://doi.org/10.11606/ issn.2176-7262.v53i4p379-388

6. Fitzmaurice MG, Wong A, Akerberg H, Avramovska S, Smithburger PL, Buckley MS, et al. Evaluation of Potential Drug-Drug Interactions in Adults in the Intensive Care Unit: A Systematic Review and Meta-Analysis. Drug Saf. 2019;42(9):1035-44.

7. Janković SM, Pejčić A V., Milosavljević MN, Opančina VD, Pešić N V., Nedeljković TT, et al. Risk factors for potential drug-drug interactions in intensive care unit patients. J Crit Care. 2018;43:1-6.

8. Guthrie B, Makubate B, Hernandez-Santiago V, Dreishculte $\mathrm{T}$. The rising tide of polypharmacy and drug-drug interactions: population database analysis 1995-2010. BMC Med. 2015;13:74. 